\title{
Mineros en movimiento: patrones residenciales y cultura sindical en perspectiva comparada ${ }^{1}$
}

\author{
Omar Manky²
}
1 Agradezco a Narda Henríquez por motivarme a presentar este trabajo, que sintetiza y actualiza varias ideas que he venido trabajando durante los últimos ańos. A los revisores ciegos, que permi- tieron que este artículo mejore sustancialmente gracias a sus comentarios, sugerencias y preguntas. A los directores de la revista y a su asistente editorial por el acompańamiento y paciencia durante todo el proceso.
2 Licenciado en Sociología por la Pontificia Universidad Católica del Perú (PUCP). Doctor y magíster en Relaciones Laborales por la Universidad de Cornell. Profesor del Departamento Aca- démico de Ciencias Sociales y Políticas de la Universidad del Pacífico. Investigador del Centro de Investigación de la Universidad del Pacífico (CIUP). Correo: wo.mankyb@up.edu.pe. https://orcid. org/0000-0003-1741-3461

Fecha de recepción: 14/01/2020. Fecha de aceptación: 29/04/2020. 


\section{Mineros en movimiento: patrones residenciales y cultura sindical en perspectiva comparada}

\section{RESUMEN}

Uno de los cambios más significativos en la industria minera a nivel global ha sido el del fin del modelo de «ciudad minera». Desde hace más de dos décadas este ha sido reemplazado por un modelo de «hotel», en el que los trabajadores duermen mientras están en el campamento, dejando a sus familias lejos del asentamiento minero por varios días. Este artículo tiene tres objetivos. Primero, colocar esta transformación en el contexto más amplio de cambios en la industria minera latinoamericana. Segundo, resumir, partiendo de mis investigaciones previas, los caminos a través de los cuales este sistema ha socavado las estrategias tradicionales del sindicalismo minero en el Perú. Por último, argumentar que, a pesar de dichas transformaciones, los trabajadores no son actores pasivos a los nuevos arreglos, sino que han desarrollado nuevas formas de acción, aunque con variaciones que dependen de los contextos locales y nacionales en los que ellas ocurren.

Palabras clave: minería, Perú, sindicatos.

\section{Miners on the Move: Residential Patterns and Labor Culture in Comparative Per- spective}

\section{Abstract}

One of the most important changes in the global mining industry has been the demise of the «mining town» model. For more than two decades it has been replaced by a «hotel» model, in which workers sleep while in the camp, leaving their families far from the mining settlement for several days. This article has three objectives. First, to put this transformation in the broader context of changes in the Latin American mining industry. Second, to summarize, based on my previous research, the ways this system has undermined the traditional strategies of Peruvian mining unions. Finally, to argue that, despite these transformations, workers are not merely passive actors in the new settings, but rather they have developed new forms of action, albeit with variations depending on the local and national contexts in which they occur.

Keywords: mining, Peru, labor unions. 


\section{INTRODUCCIÓN}

Durante las últimas dos décadas, los regímenes atípicos de trabajo se han extendido en toda América Latina (Celis Ospina, 2012; de la Garza, 2000). Estos tienen varias facetas, tanto organizativas - por ejemplo, a través de la subcontratación de trabajadores o del uso de contratos temporales- como espaciales, a través de la migración o nuevos patrones de residencia para los trabajadores. En la industria minera, uno de los cambios más importantes ha sido el del fin de la ciudad minera, hoy reemplazada por el uso de hoteles en los que los trabajadores duermen mientras están en el campamento. Cierto número de días a la semana, se les da tiempo para ir a sus domicilios, ubicados por lo general en ciudades alejadas del punto de producción (Manky, 2017b, 2017a).

Aunque aparecieron en Australia y Canadá (Sibbel, 2010; Storey, 2001), este modelo es hoy también común en países del Sur Global, como Perú o Chile. Este artículo tiene tres objetivos. Primero, explicar en qué consiste esta transformación, colocándola en el contexto más amplio de cambios en la industria minera latinoamericana. Segundo, detallar los caminos a través de los que este sistema ha socavado algunas estrategias tradicionales del sindicalismo minero, resumiendo y volviendo sobre mi trabajo anterior en este sector (Manky, 2017b, 2020). Por último, mostrar cómo, a pesar de dichas transformaciones, los trabajadores no son actores pasivos frente a los nuevos arreglos, sino que pueden dar forma a sus resultados.

Para desarrollar estos objetivos, me concentro en mi experiencia de investigación de diferentes minas en dos de los mayores productores mineros de la región: Chile y Perú (Manky, 2018, 2019). Al hacerlo, espero proporcionar luces sobre un cambio global desde una perspectiva localizada, pero también introducir la perspectiva laboral en los estudios sobre industrias extractivas en Perú, los cuales han ignorado esta mirada durante los últimos quince años.

\section{TRANSFORMACIONES EN LA INDUSTRIA MINERA LATINOAMERICANA}

\section{Un contexto de múltiples cambios}

La constitución de regímenes de «hotel minero» ha sido una transformación radical, pero no la única experimentada en la industria minera latinoamericana. Para empezar, es importante notar que estos regímenes se han vinculado a la llegada de un gran número de inversores globales a la región (Bridge, 2004). Segundo, ellos vienen acompañados por cambios en las instituciones laborales, que favorecieron el uso de empleo precario, por ejemplo a través de la subcontratación laboral, a nivel nacional (Aravena y Núñez, 2009; Manky, 2014). Tercero, el sistema ha sido adoptado en un contexto de crecientes debates en torno a la minería y el desarrollo local, 
en el que comunidades indígenas y campesinas se han movilizado para rechazar o renegociar las condiciones bajo las que la industria opera (Bebbington y otros, 2008; Dore, 2000). En esta sección hago un breve mapa de estos procesos con el objetivo de contextualizar mejor el surgimiento y principales dinámicas de los hoteles mineros.

Siguiendo un patrón común en el sur global, entre 2004 y 2014 las inversiones mineras en América Latina crecieron significativamente (Bridge, 2004; North y Grinspun, 2016). Brasil, Chile, México y Perú estuvieron entre los destinos más importantes para estas inversiones, ubicándose entre los principales productores de oro, zinc, plata y cobre. A pesar de este vertiginoso aumento, una participación masiva en el mercado global no era nueva, pues la minería ha sido casi siempre crucial en economías orientadas a la exportación, sobre todo en el caso de Chile y Perú, donde además existe una rica tradición de estudios sobre esta industria y sus efectos sociales (Bergquist, 1986; Fisher y Bonilla, 1977; Flores Galindo, 1974; Klubock, 2004; Sulmont, 1980).

Ello no implica que la forma de extracción actual sea similar a la del pasado. Si bien la región ha exportado minerales desde tiempos coloniales (Dore, 2000; Fisher y Bonilla, 1977), fue durante el siglo XIX que los estados-nación de América del Sur iniciaron un proceso de consolidación, delimitación de fronteras, establecimiento de legislación y crecimiento de su participación en la economía capitalista mundial (González Miranda, 2013). A principios del siglo XX, la mayoría de los países latinoamericanos había decidido que era crucial atraer inversores extranjeros, dada la cantidad de fondos necesarios para la mejora de minas existentes y para el inicio de nuevos proyectos. Fue en este contexto que, tanto en Chile como en Perú, empresas de propiedad estadounidense comenzaron a controlar los principales depósitos mineros. Para 1950 estas manejaban más del 70\% de la producción de cobre en ambos países ${ }^{3}$.

Esta situación cambiaría hacia la década de 1970, cuando, a raíz de la aparición y popularización de discursos nacionalistas, varios gobiernos expropiaron las minas, creando burocracias nacionales para organizar la exploración, producción y exportación. Empresas como Comibol en Bolivia, Centromin en Perú, o Codelco en Chile, controlaron no solo la mina, sino que también se dedicaron a administrar las ciudades que habían sido establecidas durante la administración norteamericana: Los servicios de transporte, recreación, salud y otros fueron asumidos por el Estado (Dinius y Vergara, 2011). Sin cambios formales en su dinámica, se reprodujo mucho del modelo de «ciudad minera» tradicional: trabajadores y sus familias

\footnotetext{
3 Ver, al respecto, los excelentes relatos de Goodsell (1974) y Finn (1998) para Perú y Chile, respectivamente.
} 
habitaban viviendas propiedad de la empresa, la cual también se encargaba de proveer de servicios básicos como salud, recreación y educación (Crawford, 1995; Zapata, 1977).

Sin embargo, la falta de experiencia en la producción minera, sobre todo en Perú, y los bajos precios de los minerales durante la década de 1980, crearon una situación insostenible en la mayoría de minas. En la década de 1980, Augusto Pinochet decidió permitir las inversiones mineras privadas en Chile, aunque el crecimiento sistemático del sector privado no se produjo hasta la década de 1990 (Agacino, González y Flores, 1998; Lagos, 1997). De modo más radical, en 1993 Alberto Fujimori inició la privatización de todas las minas peruanas (Hiba, de Echave y Ospina, 2002). Más aún, emergió nueva legislación minera, en un intento por atraer grandes inversiones internacionales, flexibilizando el acceso a la tierra y la regulación medioambiental. Desde entonces, varias compañías multinacionales como BHP Billiton, Barrick-Gold, Freeport-McMoran y Glencore-Xstrata, han administrado las minas más importantes en Perú y Chile.

Además del cambio en los patrones de propiedad minera, toda América Latina experimentó un proceso de aprobación de nuevas leyes laborales, siendo este proceso particularmente radical en Chile y Perú (Cook, 2007). En términos de derechos colectivos, se buscó evitar la negociación por rama de actividad, privilegiando, en su lugar, las negociaciones a nivel de empresa. Junto a ello se limitaron los derechos a la huelga por parte de los obreros y se permitió el despido arbitrario, así como varias formas de trabajo atípico bajo las que los trabajadores gozaban de menos derechos, como en el caso de los empleados a través de empresas de subcontratación (Uriarte y Colotuzzo, 2009).

Esta transformación es digna de mención porque en países mineros como Bolivia, Chile y Perú, los mineros se encontraban entre los primeros grupos organizados dentro de la clase obrera (Bergquist, 1986; Flores Galindo, 1974; Zapata, 2002), jugando un papel clave en la configuración de las relaciones laborales a lo largo del siglo XX (Angell, 1974; Kruijt y Vellinga, 1979). Frente a los gobiernos autoritarios de Pinochet y Fujimori, y aunque con trayectorias específicas dependientes de las tradiciones políticas de cada caso, los mineros se verían debilitados (Manky, 2019).

A pesar de ello, es importante notar que la continua relevancia de la industria minera - en Chile y Perú los minerales representaron más del 50\% de las exportaciones entre 2000 y 2014 - permitió que sus sindicatos se encuentren, todavía, entre las organizaciones de trabajadores más fuertes de cada país, en un contexto de precariedad laboral. 


\section{El hotel minero}

Es en este contexto de cambios económicos y políticos que la legislación para regular el uso de hoteles mineros fue implementada. Ello ocurrió principalmente para adaptarse a las necesidades de los inversores mundiales. Por una parte, fue necesario legalizar el uso de jornadas atípicas de trabajo, que aumentasen el tiempo máximo de trabajo diario, tal como ocurría en los países de origen de estas empresas. Esto implicaba permitir que los mineros estén hasta por doce horas en el lugar de trabajo, a cambio de tener varios días seguidos de descanso. Por otro lado, mientras la legislación anterior volvía a las empresas mineras encargadas de ofrecer alimentos, vivienda y servicios de salud a los mineros y sus familias, las nuevas leyes las liberaban de tales obligaciones. En el futuro, solamente tendrían que ofrecer alojamiento individual a aquellos empleados que trabajen en lugares aislados (respecto de estos cambios, ver la estupenda tesis de Helfgott, 2013).

La modificación legal volvió posible la popularización de los hoteles mineros. Esto se evidencia en el hecho de que ninguna empresa que haya comenzado a operar desde 1990 haya construido una ciudad bajo el modelo clásico. Incluso empresas que en algún momento apostaron por el modelo de ciudad minera derrumbaron esos espacios para dar lugar a modelos de hotel, como fue el caso de las minas en Espinar o Toquepala, en Perú, o la de Chuquicamata, en Chile. Hoy la antigua ciudad minera es un espacio arqueológico más que lleno de vida social.

Este modelo ha permitido que las familias de los trabajadores permanezcan en grandes ciudades, mientras que los trabajadores viajan a zonas alejadas de las mismas, permaneciendo en habitaciones solitarias o compartidas, aislados no solo de sus familiares sino también de las comunidades locales. Por el bajo nivel educativo en las zonas rurales, sus integrantes, especialmente en Perú, por lo general no tienen las habilidades adecuadas para encontrar un trabajo regular en una industria tecnológicamente avanzada ${ }^{4}$.

Aunque este modelo se ha utilizado extensamente en América Latina durante más de dos décadas, existe todavía un amplio espacio para discutir sus principales efectos. El grueso de investigaciones previas miró su impacto en el bienestar psicosocial de los trabajadores. Por ejemplo, hay estudios sobre el estrés (Carrasco y Vega, 2011; Garrido y Hunt, 2013), conflictos familiares (Salinas, Barrientos y Rojas, 2012) y procesos de desviación social (Rodrigo y Atienza, 2014; Villafuerte, Maldonado y Olivera, 2009). Aunque estos trabajos no comparan sus resultados con los de otras regiones, vale la pena señalar que en su mayoría han encontrado resultados

\footnotetext{
4 Es importante notar, sin embargo, que estos procesos también han sido disputados por parte de las comunidades locales, por ejemplo, al presentar reclamos respecto de la cantidad de puestos de trabajo disponibles para ellas. Al respecto, ver Manky, 2020.
} 
negativos, similares a los hallados en otros países, como Australia o Canadá (Carrington, Hogg, McIntosh y Scott, 2012; Kaczmarek y Sibbel, 2008).

Otra línea de investigación emergente se centra en los efectos del hotel minero en el desarrollo local a través de su impacto en los mercados de trabajo (Bury, 2005; De Laire, 1999). Estos estudios están preocupados por las consecuencias de las políticas de la industria minera sobre comunidades locales tales que, dada su falta de capacitación, no pueden asegurar el acceso a empleos en la industria (Himley, 2013). Del mismo modo, algunos trabajos se han centrado en cómo los hábitos de viaje de los trabajadores afectan sus patrones de gasto en las regiones mineras (Aragón y Rud, 2013; Aroca y Atienza, 2011). En general, esta corriente de investigación, más socioeconómica, es importante dado el aumento de los conflictos sociales vinculados a la industria minera, y cómo actores locales son movilizados para demandar más puestos de trabajo en las minas del país (Gil, 2009; Himley, 2013).

Estos trabajos tienen un enorme valor, pero no agotan los temas laborales. De hecho, mi investigación anterior se ha centrado en analizar, a nivel teórico (Manky, 2017a) y empírico (Manky, 2017b) los efectos de este sistema sobre los sindicatos mineros. En estos trabajos he argumentado que, aunque una mirada superficial hace pensar que los sindicatos se verían afectados — cuando no destruidos — bajo el sistema actual, el escenario es, de hecho, bastante más complejo.

Primero, porque estos cambios han sido asumidos de forma heterogénea por los trabajadores. Por una parte, en minas grandes y tecnológicamente avanzadas, he encontrado aceptación por parte de los mismos. El modelo de hotel permite que sus hijos estudien en escuelas y universidades con mayor libertad, y da mayor autonomía para que los propios mineros se dediquen a diferentes tareas económicas o políticas en sus días libres. Pero la situación es diferente para trabajadores de minas medianas o grandes, aunque con tecnologías poco avanzadas, que encuentran en la ciudad minera un régimen que todavía otorga varios beneficios. Estos trabajadores son críticos de la desaparición de las ciudades, y en algunos casos, como Marcona, incluso han sido críticos de cualquier sugerencia al respecto (Manky, 2013).

Segundo, como expongo en lo que sigue, he encontrado que los trabajadores mineros han sido capaces de renovar sus repertorios de movilización colectiva, buscando nuevas maneras de coordinar entre sí, y de ejercer presión frente al Estado y las empresas.

\section{Mineros en movimiento}

Igual que en países como Australia o Canadá, en América Latina la actividad minera tiene lugar, por lo general, en áreas alejadas de los centros urbanos más importantes. En este contexto es que las empresas tradicionalmente reclutaban a su personal de 
comunidades aledañas, fuera para asumir trabajos de poca especialización o para entrenar a los nuevos obreros (Flores Galindo, 1974; Kruijt y Vellinga, 1979). En otros casos, cuando se requería que trabajadores mejor capacitados se mudasen, esto ocurría junto a toda su familia, construyendo para este propósito ciudades administradas por la empresa (Arcienaga, 1986; Cerda, 2014).

En estos espacios, las identidades locales de la clase trabajadora se constituyeron no solamente sobre la base de su identidad ocupacional, sino también en torno a habitantes de lugares donde no solo eran obreros, sino residentes (Goodsell, 1974; Manky, 2017b). Organizados en sindicatos, los mineros no solo luchaban para mejorar sus condiciones de trabajo, sino también para conseguir que los servicios proveídos a sus familias y comunidad local fuesen adecuados (Durand, 2012; Helfgott, 2017). Historiadores del trabajo como Bergquist (1986) o Flores Galindo (1974) y sociólogos como Barrera (1978) o Zapata (1977) han documentado cómo las identidades colectivas se reproducían en los hogares y barrios, donde ellos podrían reunirse después de cada día para hablar sobre sus experiencias comunes (para una síntesis teórica, ver Manky, 2017a).

Basados en estas lógicas, podría contrastarse el potencial articulador de la ciudad minera con lo que ocurriría bajo una nueva configuración espacial. Enmarcada en una larga tradición en sociología, para la cual las ciudades mineras eran lugares de identidades de clase trabajadora de fuertes y sólidas organizaciones (Bulmer, 1975; Kerr y Siegel, 1964), la industria sería vista como una en la que, al perder su capacidad de «actuar localmente», los trabajadores verían su capacidad de acción colectiva debilitada.

En lo que sigue resumo y vuelvo sobre mi investigación previa, que da cuenta de las consecuencias del modelo de hotel sobre los sindicatos mineros (Manky, 2017b, 2017a), aunque revisándola a la luz de nuevas comparaciones entre diferentes minas. Uno de los aspectos más llamativos del modelo de hotel es que, a pesar de ser parte de una lógica global por parte de la mayoría de empresas mineras, acaba traduciéndose en prácticas específicas que dependen de diferentes factores locales y nacionales. Es por ello que el análisis que sigue tiene un énfasis comparado y nacional, primero, y luego pasa a analizar un caso en detalle.

\section{Los hoteles mineros en perspectiva nacional}

Antes de proceder con el análisis, es importante considerar la dificultad para comprobar el argumento respecto de cómo el fin de la ciudad minera se vincula con episodios de debilidad colectiva. Ello debido a que, como se ha mencionado, el paso de un modelo a otro se dio en paralelo a otras transformaciones, de orden político —en los noventas existió un gobierno autoritario y agresivo con los sindicatos- y organizativo, vinculado a cambios en la legislación laboral. Es imposible 
argumentar que es el cambio del modelo residencial el que, por sí solo, explica el declive de acciones colectivas como la huelga. La evidencia que he recolectado en diferentes trabajos que aquí sintetizo, sin embargo, me permite volver sobre la conexión entre estos fenómenos.

Gráfico 1. Número de huelgas en el sector minero peruano: 1991-2019

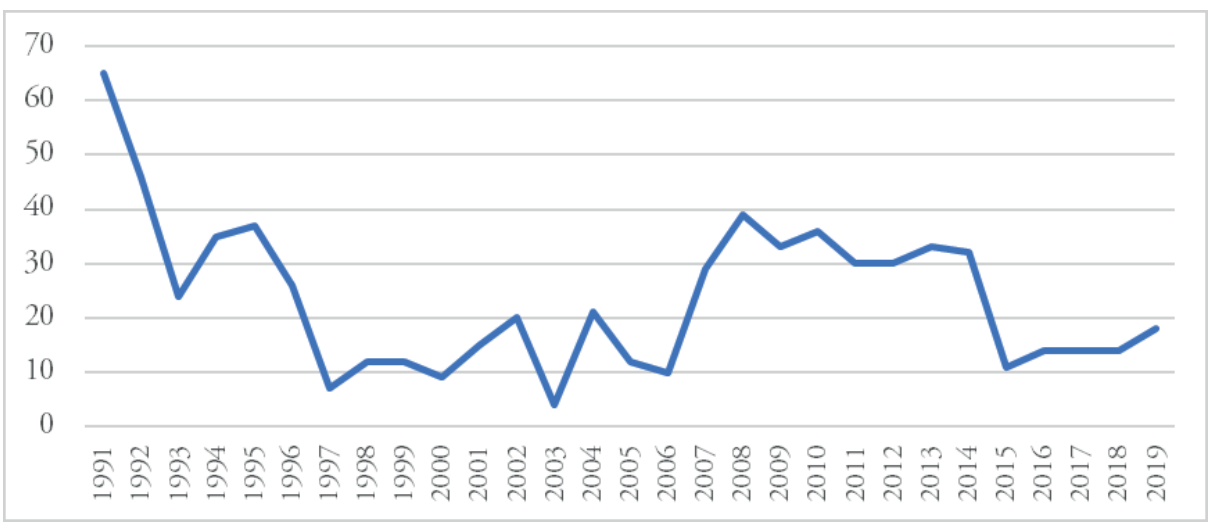

Fuente: Anuarios Laborales del Ministerio de Trabajo.

Como puede apreciarse, el número de huelgas en el sector minero sufre una caída clara durante los años noventa. Las huelgas aumentarán hacia mediados de la primera década de este siglo, llegando a su pico entre los años 2007 y 2009, los años del boom de los commodities, para volver a niveles bajos de menos de veinte huelgas anuales, desde 2015.

Existen múltiples teorías respecto de los orígenes y dinámicas de las huelgas (Dixon, Roscigno y Hodson, 2004; Hyman, 1989). En el caso minero, es importante subrayar que múltiples factores permiten entender sus dinámicas, desde la tecnología utilizada en cada mina - las condiciones de trabajo en la minería subterránea y de tajo abierto son bien distintas-, hasta la cultura organizacional de la empresa que negocia con los trabajadores. En medio de esta complejidad, sin embargo, es posible notar algunos hechos que dan cuenta de los efectos del sistema de hotel minero.

La tabla 1 agrupa a las nueve operaciones mineras peruanas que experimentaron más de diez huelgas entre 2007 y 2019. De las 333 huelgas registradas en esos trece años, 135 ocurrieron en estas minas. El dato no es menor, tomando en cuenta que para 2011 el MINEM daba cuenta de la existencia de más de 398 operaciones en todo el país ${ }^{5}$. Aunque no todas las operaciones de la tabla cuentan hoy con un

5 https://www.americaeconomia.com/negocios-industrias/peru-existen-398-unidades-mineras-enproduccion-y-100-companias-realizando-explo 
régimen de ciudad minera, todas lo tuvieron al inicio de sus operaciones: todas estas minas iniciaron operaciones antes de 1990, y aun aquellas que han pasado a un régimen de hotel, como Casapalca o Atococha, lo han hecho parcialmente, pues los trabajadores a los que entrevisté viven todavía en las áreas cercanas a la mina con sus familias, en muchos casos integrados a la comunidad local.

Tabla 1. Operaciones mineras con más huelgas (2007-2019)

\begin{tabular}{lccc}
\hline Operación (Empresa) & $\begin{array}{c}\text { Huelgas } \\
\text { 2007-2019 }\end{array}$ & $\begin{array}{c}\text { ¿Hotel } \\
\text { minero? }\end{array}$ & $\begin{array}{c}\text { Porcentaje de la PEA empleada } \\
\text { en minería en el distrito donde se } \\
\text { ubica la mina }\end{array}$ \\
\hline Marcona (Shougang) & 25 & No & $24,75 \%$ (Marcona) \\
\hline $\begin{array}{l}\text { Uchucchacua } \\
\text { (Buenaventura) }\end{array}$ & 17 & No & $40,92 \%$ (Oyón) \\
\hline $\begin{array}{l}\text { Orcopampa } \\
\text { (Buenaventura) }\end{array}$ & 16 & No & $40,71 \%$ (Orcopampa) \\
\hline $\begin{array}{l}\text { Casapalca } \\
\text { CIA Casapalca) }\end{array}$ & 15 & Si & $70,94 \%$ (Chicla) \\
\hline Atacocha (Milpo) & 14 & Sí & $42,29 \%$ (Yarusyacan) \\
\hline San Juan de Chorunga & 13 & No & $40,95 \%$ (Río Grande) \\
\hline $\begin{array}{l}\text { Argentum-Morococha } \\
\text { (Pan American Silver) }\end{array}$ & 12 & No & $51,41 \%$ (Morococha) \\
\hline Raura & 12 & No & $52,55 \%$ (San Miguel de Cuari) \\
\hline Yauliyacu & 11 & No & $70,94 \%$ (Chicla) \\
\hline
\end{tabular}

Fuente: Informes mensuales del Ministerio de Energía y Minas y Censo Nacional de 2017. Elaboración propia.

Un indicador interesante es el porcentaje de la PEA que trabajaba en minería cuando se dio el censo nacional de 2017. Mientras que a nivel nacional solamente el 1,05\% de la PEA fue empleada en explotación de minas y canteras, estos porcentajes son bastante más altos en distritos en los que tradicionalmente se ha utilizado un régimen de ciudad minera. Por ejemplo, en Oyón u Orcopampa, cerca al 40\% de la PEA estaba empleada en minería, y en Chicla el porcentaje llegaba a pasar el $70 \%$. Estas cifras contrastan con aquellas operaciones que comenzaron empleando un hotel minero. Es el caso de minas como La Arena o Yanacocha, en cuyos distritos se emplea, en minería, a menos del 5\% de la población. En estos casos se han registrado menos de dos huelgas en los últimos doce años. 


\section{La crisis de la protesta en Toquepala ${ }^{6}$}

Sería posible lograr un análisis más fino mirando específicamente las características de aquellas empresas que utilizan un régimen de hotel minero, pero dicho dato no está sistematizado en ningún ministerio. De ahí que investigar sobre los regímenes en cada mina debe hacerse sobre la base de entrevistas y trabajo de archivo.

Por ello, en mi trabajo anterior (Manky, 2017b) desarrollé una investigación sobre un caso específico, el de la mina de Toquepala. Es un caso relevante porque pasó de un modelo de ciudad a uno de hotel a mediados de los 2000. Esto facilita una comparación entre regímenes, a diferencia de un estudio centrado en empresas que hayan utilizado únicamente el sistema de hotel en las que otras variables acaban afectando cualquier comparación.

En lo que sigue resumo los hallazgos de ese trabajo, aunque releyéndolos través de múltiples tipos de comparaciones entre lo experimentado por los obreros mineros en otros espacios ${ }^{7}$. La mina de Toquepala es una de las más grandes a tajo abierto en Perú. Dado su aislamiento geográfico, cuando se construyó, a finales de la década de 1950, la compañía norteamericana encargada del proyecto reclutó trabajadores de áreas rurales distantes. Estos trabajadores fueron posteriormente como mineros, ganando estabilidad laboral y salarios por encima de la media nacional (Becker, 1983). Además, construyó una ciudad que prestaba, a trabajadores y sus familias, servicios como restaurantes, una iglesia, escuelas y espacios públicos para reunión y diversión de distinto tipo.

El campamento reflejaba las jerarquías dentro de la empresa, con diferentes barrios para gerentes y obreros. Cada grupo tenía diferentes derechos en cuanto a educación, salud y recreación (por ejemplo, canchas de fútbol para obreros y hasta de golf para la gerencia), lo que producía una fuerte identidad territorial. Al igual que en otras ciudades mineras, siendo a la vez empleador y propietario, Toquepala tenía control sobre la fuerza de trabajo más allá del punto de producción. En respuesta, el sindicato se tornó en un actor crucial en la ciudad, pues trabajadores y vecinos lo utilizaban activamente para exigir cambios en su relación con condiciones de trabajo, derechos de alojamiento o calidad de los espacios públicos.

Hasta inicios de la década de 1990, la mayoría de los procesos de negociación colectiva en Toquepala involucraron paralizaciones al interior del campamento

6 Esta sección se basa en Manky (2017b).

7 Específicamente, reuní información a través de entrevistas a obreros y visitas a tres tipos de mina: (a) las más nuevas, que siempre han organizado la producción a través de modelos de hotel minero; (b) aquellas que, a pesar de ser una minoría, aún operan bajo un régimen de empleo minero tradicional; y (c) antiguas ciudades mineras que se han desplazado del modelo clásico hacia el de hoteles mineros desde los años noventa. Este esfuerzo comparativo, que involucró más de un año de observaciones y más de cien entrevistas, permitió obtener una mirada compleja sobre el fenómeno. 
minero. Por lo general, los trabajadores llegaban a un punto en el que, para avanzar con la negociación, la huelga era crucial. Esto cambiaría parcialmente con las reformas neoliberales de Alberto Fujimori, cuando la empresa despidió a importantes dirigentes sindicales y desarrolló prácticas de subcontratación con cerca del 40\% de los trabajadores (Manky, 2017b). A pesar de lo anterior, en mi revisión de fuentes secundarias encontré evidencia acerca de la continuidad de paralizaciones, aunque más cortas. Más aún, los archivos sindicales muestran que, aún hacia finales de los noventa e inicios de 2000, las personas seguían participando en las actividades organizadas por el sindicato, e incluso en algunas huelgas al interior del campamento (Manky, 2017b).

Esta situación cambiaría cuando, a mediados de los 2000 la empresa apostó por un sistema de hotel minero. La decisión se tomó pensando en la posibilidad de ahorrar dinero, pues la administración de espacios para trabajadores y familias se había vuelto insostenible. Es importante resaltar que encontré miradas diferentes sobre la aceptación o rechazo de los trabajadores a esta medida. La mayoría de mis entrevistados indicaron que muchos trabajadores aceptaron el cambio porque les daba la posibilidad de tener una mejor calidad de escuelas para sus hijos, pero algunos, sobre todo los mayores, veían con nostalgia la pérdida de un espacio en el que podía establecerse una comunidad (el discurso sobre el pasado glorioso de la comunidad minera puede también restrearse en espacios como Marcona. Ver, al respecto, Manky, 2013).

Al margen de este cambio, los efectos del nuevo sistema, que implicaba para los obreros estar cuatro días en cuartos en la mina y cuatro en sus casas en ciudades como Lima, Tacna o Arequipa, fueron varios. El sindicato dejó de organizar demostraciones masivas alrededor de ese año. Cuando se intentó organizar nuevas huelgas, estas ocurrieron de maneras novedosas: en lugar de organizar piquetes y bloquear carreteras en el campamento, donde cada vez vivía menos gente, los trabajadores se iban a sus casas, ubicadas en diferentes ciudades del sur del país, para descansar. Desde 2007, el número de huelgas organizadas en este campamento descendió rápidamente (Manky, 2017b). La implementación de un modelo de hotel minero socavó las estrategias basadas en el lugar de trabajo y en una fuerte identidad local.

A este cambio deben ańadirse elementos como el cambio en las leyes laborales, el aumento de la subcontratación y de la cultura obrera peruana, cada vez menos centrada en la lógica del sindicalismo clasista. Sin embargo, el peso de los últimos elementos parece reducirse al comparar este caso con el de Marcona. Hasta donde he podido investigar, esta es la mina más grande entre las que todavía utiliza un modelo clásico de ciudad minera (Goodsell, 1974). Es, como se vio en la tabla 1, la mina que ha experimentado más huelgas en el país durante los últimos diez años. 
En mis visitas a este espacio (Manky, 2013, 2019) encontré también que, a pesar de las limitaciones en su liderazgo, este emerge a nivel local, dada la posibilidad que tienen los trabajadores para encontrarse y conversar sobre sus problemas.

Los mineros que dirigieron las protestas frente a esta empresa en los últimos veinte años fueron siempre personas que habían vivido de niños en la ciudad de Marcona, con padres obreros. Eran colegas, pero también amigos de barrio. Es sintomático que uno de los espacios simbólicamente más representativos de esta ciudad sea un monumento a Saúl Cantoral, dirigente minero asesinado a fines de los ochenta. Más aún, en las protestas que he podido observar, es común que los obreros sean acompañados por sus familias, en especial sus parejas, que viven también en la ciudad.

¿Cómo explicar la diferencia entre campamentos? El hotel minero parece haber producido dos cambios clave, en la relación entre trabajadores y entre estos y sus familias (Manky, 2017a, 2017b). Primero, la antigua ciudad de Toquepala parece hoy un pueblo fantasma, donde el sindicato no tiene gran capacidad de convocar a las personas espontáneamente. En Marcona es posible ver cómo, durante los cambios de turno, decenas de trabajadores van a sus casas, al local sindical o a bares en la ciudad para conversar unos minutos. Esto permite que los trabajadores estrechen lazos con la comunidad local, que en muchos casos los han apoyado en sus protestas.

Segundo, hay un cambio en las dinámicas familiares. Dispersos alrededor de diferentes puntos del país durante la mitad de la semana, las actividades privadas de los obreros ganan mayor relevancia a expensas de las relacionados con la acción colectiva. Además, escasea la participación de las familias de estos mineros, lo que contrasta con casos como los de Marcona o Casapalca, donde encontré numerosas historias de esposas que apoyaban las actividades sindicales. No es extraño ver, en sus paralizaciones, a la comunidad organizada cerca de la carretera, dispuesta a apoyar a los trabajadores.

\section{Agencia obrera, Variación local y NaCiONAL}

A pesar de este panorama, los trabajadores han podido mejorar sus condiciones de trabajo y salarios a través de la negociación colectiva, incluso después de la instalación de un modelo de hotel. El eje de mi investigación ha estado puesto principalmente en las dinámicas internas del sindicato, es decir, su capacidad de innovar estrategias, que a nivel externo, esto es, los resultados que alcanzan en el tiempo. Sin embargo, es importante notar que sindicatos como el de Toquepala han logrado seguir negociando bajo el nuevo modelo. En los últimos tres convenios (2012-2015, 2015-2018, 2018-2021), el sindicato consiguió, en coordinación con los otros sindicatos de Southern, aumentos salariales de 5\% en cada año de vigencia 
del convenio (firmados por tres años), y una bolsa de más de 40000 soles para cada afiliado, dado que el acuerdo se logró a través del trato directo con la empresa ${ }^{8}$.

¿Cómo fue esto posible, en un contexto en el que la capacidad de movilización parece haber decaído? Aquí es relevante recurrir a Herod (2001), para quien la «escala» en la que actúan los trabajadores organizados no es solo una métrica física, sino una construcción social. Desde esta perspectiva, que rescata la capacidad de agencia de las personas, no basta con indicar las instancias en las que el poder de negociación de los trabajadores se ha visto afectado, sino también reconocer su capacidad para construir nuevos espacios de acción. Cabe entonces subrayar que, aunque la capacidad de los trabajadores mineros para movilizarse localmente se ha visto afectada, estos han sido capaces de construir nuevas redes en distintos niveles.

En línea con lo que otros estudios han demostrado, los sindicatos pueden extender su influencia más allá del lugar de trabajo, produciendo así nuevas escalas de lucha (Anner, 2011; Brookes, 2013; Ellem, 2006). Las estrategias de mejora implementadas por cada sindicato variarán dependiendo de las estrategias de los empleadores, las condiciones locales, o las experiencias de movilización previas.

En el caso de Toquepala, identifiqué varios mecanismos de respuesta (Manky, 2017b), que parten de un trabajo multiescalar. Por ejemplo, aprovechando los cambios tecnológicos experimentados en los últimos diez años, se han tornado cruciales teléfonos móviles y correo electrónico para coordinar actividades. En contraste con un modelo en el que los sindicalistas vivían en la ciudad minera y enviaban comisiones especiales que costaba mantener, hoy pueden dedicarse a presionar en varios espacios al mismo tiempo. La implementación de un modelo de hotel minero también ha incentivado a los dirigentes del sindicato a buscar mejorar la coordinación con otros sindicatos que forman parte de la empresa dueña de Toquepala (que maneja también la mina de Cuajone y la refinería en Ilo). Además, el sindicato fortaleció sus redes con otros sindicatos mineros, como los de la federación nacional o hacia el extranjero.

Lo anterior sugiere una importante transformación en el sindicalismo, en la que parte de su fortaleza radica menos en su capacidad de movilizar a las bases que en acciones estratégicas por parte de los dirigentes. Parece ocurrir, entonces, que la acción colectiva se organiza más en torno a una lógica de grupo de interés que de movimiento social, paradójicamente de forma inversa a la tendencia en otras sociedades, como en los Estados Unidos (Milkman y Voss, 2004).

Esto no necesariamente implica que haya desaparecido un discurso clasista o radical en el sindicato de Toquepala. Pero sí que este parece acabar combinándose

\footnotetext{
8 https://diariocorreo.pe/edicion/moquegua/southern-peru-firma-convenios-colectivos-con-tressindicatos- 835282
} 
con dinámicas que se acercan más a un liderazgo que podría denominarse "técnico", basado en la capacidad de coordinación estratégica, más que en huelgas masivas y contenciosas, como por ejemplo sí ocurre en Marcona (para una discusión teórica sobre espacio y política organizativa, ver Manky, 2017a). Estos dirigentes pueden mantener un discurso radical, pero a este suman un amplio conocimiento de la legislación, redes sólidas hacia ONG y grupos de activistas en distintas ciudades, y la conciencia de que este conocimiento y redes es tan importante como la movilización en el campamento.

Sobre la base de mis visitas a diferentes campamentos mineros, considero que las dinámicas descritas se sostienen, aunque con variaciones que dependen de las capacidades organizacionales de cada sindicato y, por supuesto, del entorno en que actúan. En particular deben considerarse dos aspectos. Primero, que dependiendo del entorno local en el que opera la empresa, el sistema de "hotel minero" puede generar un efecto adicional: el conflicto entre trabajadores y comunidades locales. En el caso de Toquepala esto no ocurrió, pues la mina se encuentra en un espacio alejado de comunidades campesinas, y la densidad poblacional en el entorno no es alta. Sin embargo, ello no es así en espacios como el de Antamina, donde existen varias comunidades cerca al área de operaciones de la empresa, con grupos afectados por la minería y grupos interesados en trabajar para la mina (ver Manky, 2020).

En ambos casos, el modelo de hotel minero termina rompiendo los vínculos entre trabajadores y comunidad local. Pero en Antamina la comunidad local es más densa, organizada y con capacidad de articulación para exigir a la empresa. Para los comuneros, en cambio, los trabajadores pueden ser vistos o como cómplices de la contaminación que sufren o como competidores en un mercado de trabajo muy reducido. En mis visitas a Antamina o Tintaya, en Cusco, registré testimonios de miembros de comunidades locales que exigían el fin del modelo de hotel, porque, parafraseando a un entrevistado, «beneficiaba con trabajo a foráneos».

En este sentido, los trabajadores acababan deslocalizados en un sentido adicional: sin capacidad de hacer movilizaciones frente a posibles enfrentamientos con la comunidad. Esto agrega una dificultad adicional a la organización de huelgas locales. Cuando ellas ocurren en espacios como Marcona, los trabajadores pueden comer en casa o recurrir a tiendas de sus vecinos para comprar comida. Pero en espacios en los que ellos viven en hoteles administrados por la empresa, si no trabajan, o deciden salir del campamento como parte de su protesta, organizar sus comidas es más difícil. Especialmente si no cuentan con respaldo por parte de la comunidad local.

El segundo aspecto a notar es la variación institucional. Al respecto, el modelo chileno ilumina los espacios de acción de los trabajadores peruanos. De acuerdo al Código de Trabajo en el país del sur, el tiempo de trabajo semanal no puede exceder de 45 horas, distribuido a lo largo de cinco a seis días. Aunque las horas 
extras son legales en este país, ningún trabajador puede exceder las diez horas diarias. Esta legislación constriñe el uso de jornadas atípicas de trabajo y de modelos como el del hotel minero. Es por ello que desde los noventa se permite también el uso de un «régimen de excepción», bajo el cual, a través de una autorización explícita del Estado, se permite jornadas de más de diez horas diarias.

Para obtener este permiso, que además debe ser renovado cada cuatro años, las empresas deben cumplir con una serie de condiciones. En primer lugar, aun cuando excepcional, el número de días que los obreros pueden permanecer en el hotel minero está regulado, pues la ley establece que el número máximo de jornadas laborales continuas es de veinte en el caso de las actividades temporales (por ejemplo, la construcción de un campamento minero) y catorce en el caso de los trabajos permanentes. Segundo, deben mostrar que el uso de tiempo de trabajo ordinario es poco práctico por los procedimientos técnicos empleados en la empresa, y por el aislamiento de las operaciones. Tercero - y esto es crucial — se requiere una aprobación explícita por parte de los trabajadores, representados por un sindicato o, si no existiese, por un comité autónomo. Por último, el diseño del alojamiento minero debe también sujetarse a estándares básicos, vinculados a sus condiciones de electricidad, suelos, paredes, y la temperatura en cada dormitorio.

Esta situación contrasta con el caso peruano, en el que, como en Chile, el trabajo regular diario no puede ir más allá de ocho horas, ni 48 horas en una semana de trabajo regular. Del mismo modo, también se permite, desde la Ley de Trabajo del gobierno de Fujimori, el uso de horarios excepcionales. Pero las directrices no son tan específicas como en el caso chileno, pese a que se indica que ella no aplica a todo tipo de negocios, sino solamente a aquellos en los que el uso de un horario típico afectaría negativamente la eficiencia de la empresa.

Fue solamente en 2006 cuando, debido a las constantes quejas de los trabajadores peruanos, se decidió especificar las condiciones bajo las que se podía usar un sistema de hotel minero. Desde el Tribunal Constitucional se indicó que, para avanzar con esta forma de organización del trabajo, las empresas debían realizar una «prueba de protección». En ella se indica, por ejemplo, que los trabajadores no pueden estar durante más de tres semanas continuas en el campamento, y que las empresas están obligadas a proporcionar «sistemas adecuados de seguridad, salud, alimentación y descanso» a los empleados. Es llamativo, sin embargo, que no se den datos específicos respecto de qué sería exactamente "condiciones adecuadas», dejándolo a la libre interpretación de la empresa, trabajadores y poder judicial. El código minero peruano no detalla las condiciones «adecuadas» de un hotel minero, en comparación no solamente con el modelo chileno, sino incluso con la legislación que regulaba el uso de ciudades mineras durante el siglo XX. Como es de esperar, esto genera una considerable variación en los estándares entre diferentes minas (Chávez, 2010). 
Más importante aún, en contraste con el caso chileno, en este no se requiere un acuerdo entre empresa y trabajadores, ni se requiere, antes de iniciar un modelo de hotel, la aprobación explícita del Estado. En este contexto, las empresas que operan en el Perú tienen un mayor nivel de negociación respecto a trabajadores y Estado en la implementación de modelos de hotel minero.

De este modo, aunque el análisis sobre los efectos de este modelo aplica en líneas generales a ambos países, diferencias institucionales crean mejores condiciones para las estrategias colectivas de los trabajadores en Chile. Es llamativo que en este caso las jornadas de trabajo se discutan durante el proceso de negociación colectiva, convirtiéndose en un punto de influencia para los sindicatos. Dado que hay espacio para mejorar las condiciones de trabajo de los mineros a cambio de aceptar el uso de hoteles mineros de las compañías, el régimen de hotel podría convertirse en una herramienta para negociaciones más equilibradas entre trabajo y capital.

\section{Conclusiones}

El objetivo principal de este artículo fue resumir algunos de los efectos del cambio en la organización del trabajo minero en América Latina. Basado en mi trabajo anterior, comencé mostrando que el del hotel minero no es una política aislada, sino que se encuentra inmersa en una serie de cambios globales por parte de la industria, y nacionales, a través de políticas que buscan atraer inversores a través de reformas laborales.

En segundo lugar, propuse que el pesimismo de estudios previos sobre este cambio está parcialmente fundado, pero también argumenté, partiendo de mis trabajos previos sobre el tema, un análisis matizado. Específicamente, busqué mostrar cómo cambian y se reconstituyen espacios locales y escalas nacionales. A través de este análisis, el artículo mostró que los obreros mineros todavía pueden mejorar sus condiciones de trabajo a través de los sindicatos, pero sobre la base de una renovación en sus estrategias.

Finalmente, mostré que es necesario que todo estudio interesado en los efectos de transformaciones organizativas como la estudiada aquí sean sensibles a la manera como las mismas acaban siendo implementadas. Ningún espacio está vacío de historia y relaciones de poder, por lo que los actores locales o las legislaciones nacionales sobre las que se acaba imponiendo un modelo de hotel pueden acabar afectando su desarrollo.

Es importante notar que estos resultados son una fotografía de procesos en constante cambio. En el caso minero, el contexto en el que estudié a los sindicatos fue uno de crecimiento económico, principalmente. En un momento en el que el precio de los minerales ha caído sostenidamente, valdría la pena ver si las dinámicas 
presentadas se mantienen. De modo similar, la presión de las comunidades sobre las empresas mineras para que recluten mano de obra local podría originar cambios sustantivos sobre la organización obrera. Pero se requieren más estudios sistemáticos de casos que nacen bajo un modelo de hotel minero y se van readaptando a la presión local.

Desde una perspectiva analítica, es crucial desarrollar más investigaciones que atiendan la compleja relación entre migración laboral, desarrollo económico y estrategias sindicales. Estas investigaciones podrían iluminar mejor las estrategias no solo de los trabajadores, sino también las políticas empresariales e incluso las estrategias de las comunidades locales. Es posible imaginar estudios que partan de una perspectiva sensible a la movilidad espacial y los patrones residenciales para entender las dinámicas de diferentes trabajadores, como quienes migran de otros países y se ubican en el sector de ventas ambulantes, o quienes se dedican a trabajar con aplicaciones, recorriendo la ciudad para llevar comida o pasajeros.

Además, se necesitan estudios comparativos, tanto dentro de América Latina como entre países de esta región con otros países mineros, como Canadá o Australia. Esto informaría mejor el uso de políticas públicas, por ejemplo, respecto del empleo local o sobre los efectos familiares del modelo de hotel minero.

Mientras termino de corregir este texto, Perú enfrenta la mayor crisis económica y de salubridad de las últimas décadas. Uno de los elementos más llamativos de la misma fue el apoyo, por parte del gobierno, a la gran minería, que continuó operando a pesar de la cuarentena decretada desde mediados de marzo (Vila y Bebbington, 2020). Antamina, una de las empresas más mineras más importantes del país, detectó 210 casos de trabajadores positivos por Covid-199. Más allá de las implicancias políticas del hecho, y de la reacción del sindicato al mismo, es importante notar que necesitamos comprender los patrones de movilidad en los mercados de trabajo mineros para pensar en los efectos de hechos como este.

\section{REFERENCIAS}

Agacino, R., González, C. y Flores, J. (1998). Capital transnacional y trabajo: el desarrollo minero en Chile. Santiago: LOM.

Angell, A. (1974). Partidos politicos y movimiento obrero en Chile. CDMX: Era.

Anner, M. (2011). Solidarity Transformed: Labor responses to Globalization and Crisis in Latin America. Nueva York: Cornell University Press. https://doi. org/10.7591/9780801460579

\footnotetext{
9 https://gestion.pe/economia/empresas/antamina-revela-que-hay-210-casos-de-coronavirus-entresus-trabajadores-noticia/
} 
Aragón, F. M. y Rud, J. P. (2013). Natural resources and local communities: evidence from a Peruvian gold mine. American Economic Journal: Economic Policy, 5(2), 1-25. https://doi.org/10.1257/pol.5.2.1

Aravena, A. y Núñez, D. (2009). El renacer de la huelga obrera en Chile. Santiago de Chile: Ocho Libros.

Acienaga, R. (1986). Los mineros de la Southern Peru Copper Corporation, 1968-1981. Lima: PUCP.

Aroca, P. y Atienza, M. (2011). Economic implications of Long Distance Commuting in the Chilean Mining Industry. Resources Policy, 36(3), 196-203. https://doi. org/10.1016/j.resourpol.2011.03.004

Barrera, M. (1978). El conflicto obrero en el enclave cuprífero chileno. Revista Mexicana de Sociología, 40(2), 609-682. https://doi.org/10.2307/3539727

Bebbington, A., Humphreys Bebbington, D., Bury, J., Lingan, J., Muñoz, J. P. y Scurrah, M. (2008). Mining and social movements: struggles over livelihood and rural territorial development in the Andes. World Development, 36(12), 2888-2905. https:// doi.org/10.1016/j.worlddev.2007.11.016

Becker, D. (1983). The New Bourgeoisie and the Limits of Dependency: Mining, Class, and Power in "Revolutionary» Peru. Princeton, NJ: Princeton University Press. https:// doi.org/10.1515/9781400853236

Bergquist, C. (1986). Labor in Latin America. Palo Alto, CA: Stanford University Press.

Bridge, G. (2004). Mapping the bonanza: geographies of mining investment in an era of neoliberal reform. The Professional Geographer, 56(3), 406-421.

Brookes, M. (2013). Varieties of Power in Transnational Labor Alliances An Analysis of Workers' Structural, Institutional, and Coalitional Power in the Global Economy. Labor Studies Journal, 38(3), 181-200. https://doi.org/10.1177/0160449X13500147

Bulmer, M. I. A. (1975). Sociological Models of Mining Community. The Sociological Review, 23(1), 61-92. https://doi.org/10.1111/j.1467-954X.1975.tb00518.x

Bury, J. (2005). Mining mountains: neoliberalism, land tenure, livelihoods, and the new Peruvian mining industry in Cajamarca. Environment and Planning A, 37(2), 221239. https://doi.org/10.1068/a371

Carrasco, C. y Vega, P. (2011). Una aproximación a las condiciones de trabajo en la gran mineria de altura. Santiago de Chile: Dirección de Trabajo.

Carrington, K., Hogg, R., McIntosh, A. y Scott, J. (2012). Crime Talk, the Mining Investment Pipeline. FIFO and Frontier. Cultural Conflict, 53, 1-14.

Celis Ospina, J. C. (2012). La subcontratación laboral en América Latina: Miradas multidimensionales. Medellín: CLACSO/ Escuela Sindical.

Cerda, R. (2014). La masacre de El Salvador. Santiago: Cerda.

Chávez, O. N. (2010). Influencia de las jornadas laborales atipicas en accidentes de trabajo y enfermedades ocupacionales en la actividad minera. Lima: UNMSM.

Cook, M. L. (2007). Politics of Labor Reform in Latin America. University Park, PA: Penn State Press. 
Crawford, M. (1995). Building the workingmans paradise: The design of American company towns. Londres: Verso.

De la Garza, E. (2000). La flexibilidad del trabajo en América Latina. En E. de la Garza (ed.), Tratado Latinoamericano de Sociología del Trabajo (pp. 148-173). CDMX: FLACSO, FCE, UAM.

De Laire, F. (1999). La trama invisible o los claroscuros de la flexibilidad. Santiago: Dirección del Trabajo.

Dinius, O. J. y Vergara, A. (2011). Company Towns in the Americas: Landscape, Power, and Working-Class Communities. Athens: University of Georgia Press.

Dixon, M., Roscigno, V. y Hodson, R. (2004). Unions, Solidarity, and Striking. Social Forces, 83(1), 3-33. https://doi.org/10.1353/sof.2004.0107

Dore, E. (2000). Environment and society: Long-term trends in Latin American mining. EnvironmentandHistory, 6(1), 1-29.https://doi.org/10.3197/096734000129342208

Durand, A. (2012). De mineros a indígenas. En P. Davolos (ed.), El mundo del trabajo en América Latina. Tendencias y resistencias (pp. 117-166). Buenos Aires: CLACSO.

Ellem, B. (2006). Scaling labour Australian unions and global mining. Work, Employment \& Society, 20(2), 369-387. https://doi.org/10.1177/0950017006064275

Finn, J. L. (1998). Tracing the veins: Of copper, culture, and community from Butte to Chuquicamata. Berkeley: University of California Press. https://doi.org/10.1525/ california/9780520211360.001.0001

Fisher, J. R. y Bonilla, H. (1977). Minas y mineros en el Perú colonial, 1776-1824. Lima: IEP. Flores Galindo, A. (1974). Los mineros de la Cerro de Pasco, 1900-1930. Lima: PUCP.

Garrido, L. y Hunt, N. (2013). Organización del trabajo y estrés en trabajadores de la industria minera de Chile. Ciencia \& Trabajo, 15(47), 47-56. https://doi.org/10.4067/ S0718-24492013000200003

Gil, V. (2009). Aterrizaje minero: cultura, conflicto, negociaciones y lecciones para el desarrollo desde la minería en Ancash, Perú. Lima: IEP.

González Miranda, S. (2013). Las políticas salitraras peruana y chilena. ¿Del monopolio estatal a la libertad económica? (1873-1994). Cuadernos de Historia, 38, 39-77. https://doi.org/10.4067/S0719-12432013000100002

Goodsell, C. T. (1974). American corporations and Peruvian politics. Cambridge, MA: Harvard University Press.

Helfgott, F. (2013). Transformations in Labor, Land and Community: Mining and Society in Pasco, Peru, 20th Century to the Present. University of Michigan: PhD Thesis.

Helfgott, F. (2017). La persistencia de lo social: Los convenios laborales y los convenios comunidad-empresa en las zonas mineras de la Sierra Central. En O. Manky (ed.), Trabajo y Sociedad. Estudios sobre el mundo del trabajo en el Perú (pp. 84-103). Lima: PUCP.

Hiba, J. C., De Echave, J. y Ospina, E. (2002). Condiciones de trabajo, seguridady salud ocupacional en la minería en el Perú. Lima: OIT/ETM para los Países Andinos.

Himley, M. (2013). Regularizing extraction in Andean Peru: mining and social mobilization in an age of corporate social responsibility. Antipode, 45(2), 394-416. https:// doi.org/10.1111/j.1467-8330.2012.01001.x 
Hyman, R. (1989). Strikes. Londres: Springer. https://doi.org/10.1007/978-1-349-19819-1 Kaczmarek, E. y Sibbel, A. M. (2008). The psychosocial well-being of children from Australian military and fly-in/fly-out (FIFO) mining families. Community, Work \& Family, 11(3), 297-312. https://doi.org/10.1080/13668800801890129

Kerr, C. y Siegel, A. (1964). The interindustry propensity to strike: an international comparison. En C. Kerr (ed.), Labor and Management in Industrial Society (pp. 105-147). Garden City, NY: Anchor Books, Doubleday.

Klubock, T. M. (2004). Class, community, and neoliberalism in Chile. En P. Winn (ed.), Victims of the Chilean Miracle (pp. 209-260). Durhem, NC: Duke University Press. https://doi.org/10.1215/9780822385851-006

Kruijt, D. y Vellinga, M. (1979). Labor relations and multinational corporations: the Cerro de Pasco Corporation in Peru. https://doi.org/http://www.refworks.com/refworks2/?r=r eferences|MainLayout::init\#

Lagos, G. (1997). Developing national mining policies in Chile: 1974-1996. Resources Policy, 23(1), 51-69. https://doi.org/10.1016/S0301-4207(97)00008-1

Manky, O. (2013). Recordando al patrón: memoria organizacional y conflictos laborales. El caso de una empresa minera peruana. En G. Portocarrero (ed.), Sombras coloniales y globalización en el Perú de hoy (pp. 243-254). Lima: PUCP.

Manky, O. (2014). Democracia, crecimiento económico y sindicalismo en el Perú del siglo XXI. Continuidades y rupturas. Revista Latinoamericana de Estudios de Trabajo, 19(31), 195-228.

Manky, O. (2017a). El lugar importa: efectos de la movilidad en las estrategias sindicales. Revista Mexicana de Sociología, 79(1), 35-63.

Manky, O. (2017b). From Towns to Hotels: Changes in Mining Accommodation Regimes and Their Effects on Labour Union Strategies. British Journal of Industrial Relations, 55(2). https://doi.org/10.1111/bjir.12202

Manky, O. (2018). Resource Mobilisation and Precarious Workers' Organisations: An Analysis of the Chilean Subcontracted Mineworkers' Unions. Work, Employment and Society, 32(3). https://doi.org/10.1177/0950017017751820

Manky, O. (2019). Liderazgos precarios: organización y líderes sindicales en perspectiva comparada. Latin American Research Review, 54(4). https://doi.org/10.25222/larr.160

Manky, O. (2020). The end of mining labor struggles? The changing dynamics of labor in Latin America. The Extractive Industries and Society. https://doi.org/10.1016/j. exis.2020.07.007

Milkman, R. y Voss, K. (2004). Rebuilding labor: Organizing and organizers in the new union movement. Ithaca, NY: Cornell University Press.

North, L. L. y Grinspun, R. (2016). Neo-extractivism and the new Latin American developmentalism: the missing piece of rural transformation. Third World Quarterly, 37, 1-22. https://doi.org/10.1080/01436597.2016.1159508

Rodrigo, L. M. y Atienza, M. (2014). Migración y representaciones regionales: discursos sobre la Región de Antofagasta. EURE, 40(120), 159-181. https://doi.org/10.4067/ S0250-71612014000200008 
Salinas, P., Barrientos, J. y Rojas, P. (2012). Discursos sobre la discriminación de género en los trabajadores mineros del norte de Chile. Atenea, 505, 139-158. https://doi. org/10.4067/S0718-04622012000100007

Sibbel, A. M. (2010). Living FIFO: the experiences and psychosocial wellbeing of Western Australian fly-inlfly-out employees and partners. Edith Cowan University. http:// ro.ecu.edu.au/cgi/viewcontent.cgi?article $=1132 \&$ context=theses

Storey, K. (2001). Fly-in/Fly-out and Fly-over: mining and regional development in Western Australia. The Australian Geographer, 32(2), 133-148. https://doi. org/10.1080/00049180120066616

Sulmont, D. (1980). Historia del movimiento obrero minero metalurgico (hasta 1970). Lima: Tarea.

Uriarte, O. E. y Colotuzzo, N. (2009). Descentralización, tercerización, subcontratación. Lima: OIT.

Vila, G. y Bebbington, A. (2020). Political Settlements and the Governance of COVID-19: Mining, Risk, and Territorial Control in Peru. Journal of Latin American Geography. https://doi.org/10.1353/lag.0.0135

Villafuerte, M., Maldonado, H. y Olivera, G. (2009). Percepción de la población sobre la contribución de la gran minería a la economía de la ciudad de Huaraz, 2008. Aporte Santiaguino, 2(2), 291-300. https://doi.org/10.32911/as.2009.v2.n2.408

Zapata, F. (1977). Enclaves y sistemas de relaciones industriales en América Latina. Revista Mexicana de Sociología, 39(2), 719-731. https://doi.org/10.2307/3539782

Zapata, F. (2002). Los mineros como actores sociales y políticos en Bolivia, Chile y Perú durante el siglo XX. Estudios Atacameños, 22, 91-103. https://doi.org/10.4067/ S0718-10432002002200006 